\title{
Wearable Quarter-Wave Folded Microstrip Antenna for Passive UHF RFID Applications
}

\author{
Thomas Kaufmann, ${ }^{1,2}$ Damith C. Ranasinghe, ${ }^{2}$ Ming Zhou, ${ }^{2}$ and Christophe Fumeaux ${ }^{1}$ \\ ${ }^{1}$ School of Electrical and Electronic Engineering, The University of Adelaide, Adelaide, SA 5005, Australia \\ ${ }^{2}$ The Auto-ID Laboratory, The University of Adelaide, Adelaide, SA 5005, Australia \\ Correspondence should be addressed to Thomas Kaufmann; thomaska@eleceng.adelaide.edu.au
}

Received 10 August 2012; Revised 7 May 2013; Accepted 26 May 2013

Academic Editor: Charles Bunting

Copyright (C) 2013 Thomas Kaufmann et al. This is an open access article distributed under the Creative Commons Attribution License, which permits unrestricted use, distribution, and reproduction in any medium, provided the original work is properly cited.

\begin{abstract}
A wearable low-profile inset-fed quarter-wave folded microstrip patch antenna for noninvasive activity monitoring of elderly is presented. The proposed antenna is embedded with a sensor-enabled passive radio-frequency identification (RFID) tag operating in the ultra-high frequency (UHF) industrial-scientific-medical (ISM) band around $900 \mathrm{MHz}$. The device exhibits a low and narrow profile based on a planar folded quarter-wave length patch structure and is integrated on a flexible substrate to maximise comfort to the wearer. An extended ground plane made from silver fabric successfully minimises the impact of the human body on the antenna performance. Measurements on a prototype demonstrate a reflection coefficient $\left(S_{11}\right)$ of $-30 \mathrm{~dB}$ at resonance and a $-10 \mathrm{~dB}$ bandwidth from $920 \mathrm{MHz}$ to $926 \mathrm{MHz}$. Simulation results predict a maximum gain of $2.8 \mathrm{dBi}$. This is confirmed by tag measurements where a 4-meter read range is achieved using a transmit power of $30 \mathrm{dBm}$, for the case where the passive wearable tag antenna is mounted on a body in a practical setting. This represents an almost $40 \%$ increase in read range over an existing dipole antenna placed over a $10 \mathrm{~mm}$ isolator layer on a human subject.
\end{abstract}

\section{Introduction}

The miniaturisation of sensors and wireless systems is generating an explosive growth in body-centric wireless computing applications based on wearable electronic devices, especially in healthcare [1-6]. Consequently, the need for lightweight, low-profile, low cost, and wearable antennas has also grown rapidly during recent years. In this paper we present a successful design of such a wearable antenna for an emerging class of sensor-enabled passive radio-frequency identification (RFID) tags for use in healthcare applications.

The wireless identification and sensing platform (WISP) [7] is a passive RFID-based platform suitable for wireless wearable applications. In the present case, it integrates an accelerometer and a passive RFID tag into a batteryless system with the capability of being integrated into clothing. The intended application is the monitoring of high falls risk activities for elderly in hospitals and residential care facilities $[1,2]$. Typical WISP devices are currently built using a dipole antenna on a FR4 substrate and as a result exhibit poor performance when worn in close proximity to a human body.
In this case, the read range of the WISP is less than $2.5 \mathrm{~m}$ once attached to clothing worn by a person. This read range is inadequate for monitoring an elderly subject in a care facility. Furthermore, the thin dipole antenna on the WISP is fragile and can pose a significant discomfort to the wearer of the device while possible breakages can cause injury. Thus, a new antenna design is proposed to replace the dipole structure.

A few researchers have investigated wearable RFID tag antennas in the ultra-high frequency (UHF) range [8-11]. Most designs have been based on half-wave length dimensions, which correspond to approximately $16 \mathrm{~cm}$ in length at $923 \mathrm{MHz}$. In order to further reduce the physical dimensions, approaches including tapering in a bow-tie [9], meandering, [10], and meandering with a superstrate [11] have been considered. Further size reductions are achieved in different geometries using, for example, Planar Inverted F Antennas (PIFAs) [12], metamaterial superstrates [13], and folded shorted patches [14]. These designs usually use coaxial probe feeding mechanisms. However, in the context of wearable antennas, coaxial line fed antennas are (i) not easy to fabricate; 
(ii) fragile since wire connections are likely to fail or break in a wearable application using a flexible substrate; and (iii) impractical because of the difficulties of feeding the balanced two-port WISP tag circuitry.

Currently, a major development for WISP antenna is focused on applying paper and Inkjet printing to lower the fabrication cost of standard designs while retaining acceptable performance [8]. In this paper we present a different geometry of a low profile antenna meeting stringent requirements to support wearability in WISP-based applications. This is achieved by applying a microstrip inset-feeding method in a quarter-wave folded patch antenna on a flexible substrate. The increased performance of our design improves the read range for a body worn WISP. In particular, the proposed approach delivers improved performance and low profile without sacrificing efficiency. It is also worth noting that the tag does not require high permittivity materials, which helps in satisfying the substrate flexibility requirement and reduces the cost of production. Furthermore, we also demonstrate a design procedure for integrating the proposed antenna design to the 2-port WISP chip without an external balun and thus greatly simplifying the production of the antenna and reducing cost, while enhancing wearability. To the best of our knowledge, this is the first wearable antenna design for a WISP.

The remaining sections of the paper are organised as follows Section 2 presents a brief overview of RFID technology, with the aim of introducing the performance metrics relevant for the analysis; Section 3 describes the antenna requirements and the proposed design with a parametric study of the antenna to justify the dimension selection and demonstrates the ability of the antenna to be tuned to a different input impedance; Section 4 describes the simulation studies of the antenna performance in the vicinity of a wearer; Section 5 presents experimental results validating the simulation results as well as practical measurements of tag antenna performance; and finally, Section 6 concludes this paper.

\section{RFID Primer}

Radio frequency identification (RFID) is a wireless technology capable of contactless and automatic identification of individual objects or people [1] without line of sight requirements.

2.1. RFID Systems. RFID systems can be categorised into three primary components: tags, readers, and back-end systems. Figure 1 demonstrates the basic structure of a RFID system.

2.1.1. Tags. Tages also called labels, contain a microchip controller that stores unique identifying information of the object or person to which the tag is attached and an antenna for communicating the information via radio waves [9]. When a tag is placed in a radio frequency $(\mathrm{RF})$ field, it reflects back the information to the reader (modulated backscatter). Tags can be categorised into three types in terms of powering. Semipassive tags have an on-board power source and may have on-board sensors. Active tags have an on-board power supply

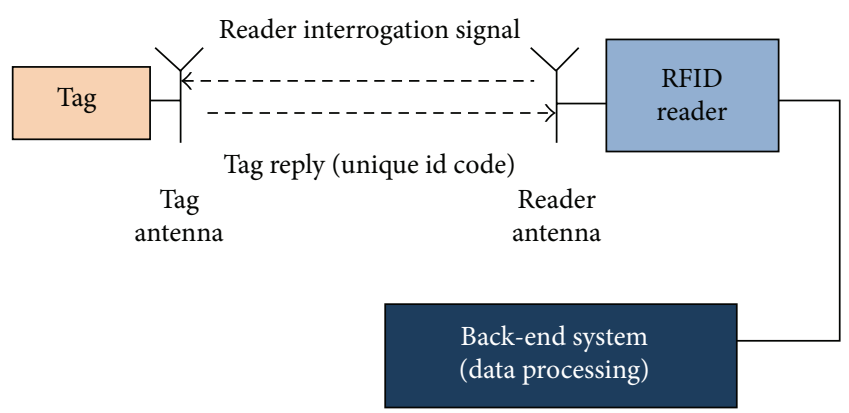

FIGURE 1: Basic RFID system block.

and also include an active receiver and a distinct active transmitter and may talk with each other and form a network. Passive tags do not have their own power supply and therefore have infinite life time as they obtain power from the incident electromagnetic (EM) radiation provided by the reading system.

2.1.2. RFID Readers. Readers are devices that communicate with the individual tags to interrogate and power them. The readers are usually distributed in fixed locations chosen to maximise the coverage of a given area. Modern RFID readers are capable of reading multiple tags (several hundred) at the same time.

2.1.3. Back-End Systems. A network of computers is connected to the readers to collect, process, and store data gathered in the interrogation process.

2.2. Tag Antenna Performance Metrics. While key characteristic measures exist for measuring the performance of antennas (such as radiation efficiency), there are a number of additional performance measures that must be investigated to ensure performance of an antenna design in a practical RFID application. In this paper we have used the following metrics: (i) received signal strength indicator (RSSI), (ii) read rate, and (iii) read range as described below.

2.2.1. Receive Signal Strength Indicator. The RSSI indicates the power level of the backscattered tag response detected by the reader. Therefore, a larger RSSI value is indicative of a stronger received signal from the WISP. The power of an RF signal received by a monostatic reader antenna can be given as [15]

$$
\mathrm{RSSI}=P_{t} G_{t}^{2} G_{\text {path }}^{2} K .
$$

In this equation, $P_{t}$ is the output power of the reader, $G_{t}$ is the gain of the reader antenna, $K$ is the WISP backscatter gain, and $G_{\text {path }}$ is the path gain of the deterministic multipath channel,

$$
G_{\text {path }}=\left(\frac{\lambda}{4 \pi d_{o}}\right)^{2}|H|^{2},
$$

where $d_{o}$ is the direct path length and $H$ is the channel response due to multipath. As visible in (1) and (2), the RSSI 
TABLE 1: Antenna requirements and specifications.

\begin{tabular}{ll}
\hline $\begin{array}{l}\text { Operational } \\
\text { frequency band }\end{array}$ & 920-926 MHz ISM band in Australia (regulated by Australian Communications and Media Authority). \\
\hline Impedance & $\begin{array}{l}\text { The WISP has a measured input impedance of } 78+16 j \Omega \text { after an on-chip matching network. A good } \\
\text { impedance matching is crucial for maximum performance. }\end{array}$ \\
\hline & $\begin{array}{l}\text { The tag should have a suitable read range to properly track the human. The current dipole has a read range of } \\
2.5 \mathrm{~m} ; \text { it is desired to increase this range. It is also desirable to have a low directivity, that is, semidirectional } \\
\text { pattern, for good angular coverage. }\end{array}$ \\
\hline Materials & The antenna must be flexible so that it can be subject to bending and can be worn by a human. \\
\hline Reliability & The tag should not be easily detuned because of its location on or near the human body. \\
\hline
\end{tabular}

is inversely proportional to the quadruple power of distance and can be used as a measure to compare the relative performance of tags operating at various reader transmit power levels and reading distances.

2.2.2. Read Rate. Upon interrogation by a reader, an RFID tag replies a unique identification code such as an electronic product code or EPC. The tag read rate is the number of successful tag reads per second as obtained by the RFID reader over the entire frequency hopping spectrum, for example, $920 \mathrm{MHz}-926 \mathrm{MHz}$ ISM band in Australia.

2.2.3. Read Range. The read range is the maximum line of sight distance from a reader antenna to the WISP tag for which successful interrogation can be achieved. It can be defined both in a real environment or a reflection free environment such as an anechoic chamber.

Based on the Friis transmission equation (3) [7], the theoretical maximum read range can be expressed in $\mathrm{dB}$ according to

$$
P_{r}=P_{t}-20 \log \left(\frac{4 \pi d_{o}}{\lambda}\right)+G_{t}+G_{r}-L_{p}
$$

In (3), the free space wavelength is $\lambda=325 \mathrm{~mm}$ at the centre frequency of $923 \mathrm{MHz}$; the power transmitted by the reader is $P_{t}=1, \mathrm{~W}=30 \mathrm{dBm}$, and $G_{t}$ and $G_{r}$ are the gain of the reader antenna and the tag antenna, respectively. The power required to turn on the WISP chip is $P_{r}=-9.5 \mathrm{dBm}$ [7] and $L_{p}$ is the polarisation loss. Evaluating the theoretically possible range sets an upper bound on the expected values for read range measurements. These computations aid in the design of the overall system.

\section{Antenna Design}

The antenna design aims at converting the WISP to a wearable WISP ( $\left.\mathrm{W}^{2} \mathrm{ISP}\right)$. The term WISP generally refers to a completely passive RFID tag supporting EPC Class 1 Generation II RFID protocol [7]. It is a sensor-enabled and programmable tag realisation that has been demonstrated for applications in human activity recognition $[1,2]$ when worn over the chest (above the sternum). However, one key issue remains the wearability of the tag [1].

An essential requirement for a wearable RFID-based monitoring system is to ensure the comfort of the subject to which the tag is attached, for example, patients in acute care or elderly in residential care. This is especially important for elderly patients in a prone position during sleep. In this case, the centre chest position has been identified as most appropriate, as an object placed on the sternum will likely have a minimal negative impact on the wearer's comfort. Hence, the size of the antenna must be restricted into a space of $35 \mathrm{~mm} \times 90 \mathrm{~mm} \times 5 \mathrm{~mm}$ and the construction materials must be flexible and soft. The design requirements for the antenna to develop a wearable and passive human activity monitoring device are summarised in Table 1.

3.1. Antenna Structure. Figure 2 shows the antenna structure. In order to limit the area occupied by the antenna to the sternum of a human body, the ground plane is limited to a width of $35 \mathrm{~mm}(0.11 \lambda)$ and a length of $83 \mathrm{~mm}(0.255 \lambda)$. Given the very restrictive constraints from Table 1, we have chosen to design a folded inset-fed microstrip patch structure with effective length of $0.25 \lambda(L=79 \mathrm{~mm})$. Folding the patch can be interpreted as the introduction of a metallic wall symmetry in the centre of a microstrip antenna. This effectively reduced the length in half compared to the original dipole design.

In the design of the folded microstrip antenna, the choice of substrate is important in terms of antenna performance. The radiation efficiency of a patch antenna depends strongly on the electrical properties of the substrate. A thicker substrate with lower dielectric constant has better efficiency, increased bandwidth, and antenna gain at the cost of increased size [16]. The substrate selected in the present design is a highly flexible Cuming Microwave C-Foam PR4 , which is a closed-cell low loss flexible foam. The relative permittivity is $\varepsilon_{r}=1.06$ and the loss tangent is $\tan \delta=0.0001$. The height is chosen as $h=3.2 \mathrm{~mm}$ as a compromise between wearability and performance. The exceptional flexibility and softness of this material are of paramount importance considering the comfort and safety of the antenna wearers.

Due to the small width of the antenna, the input impedance is very high at the first edge of the resonator on the feed side. This can be addressed using a rather long inset feed. The inset-feed point $\left(y_{0}\right)$ is $13 \mathrm{~mm}$ away from the shortened edge, which denotes the effective center of the patch. Using such a low-permittivity substrate, a relatively wide microstrip line of $8 \mathrm{~mm}$ would be required to obtain $78 \mathrm{Ohms}$ characteristic impedance. This would be too wide to efficiently feed the antenna which has a specified maximum width of $30 \mathrm{~mm}$. Furthermore, slots with a nonneglectable width have to be incorporated to allow for the inset feed. This is not practical, 


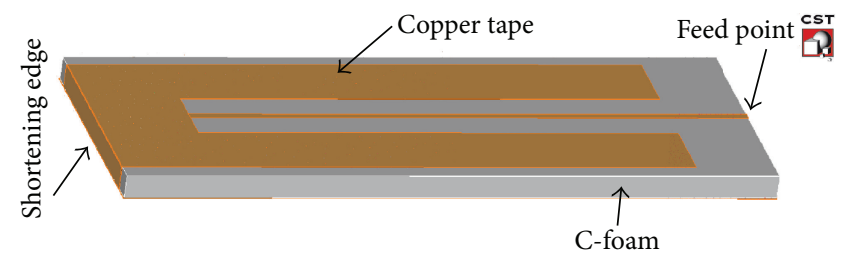

(a)

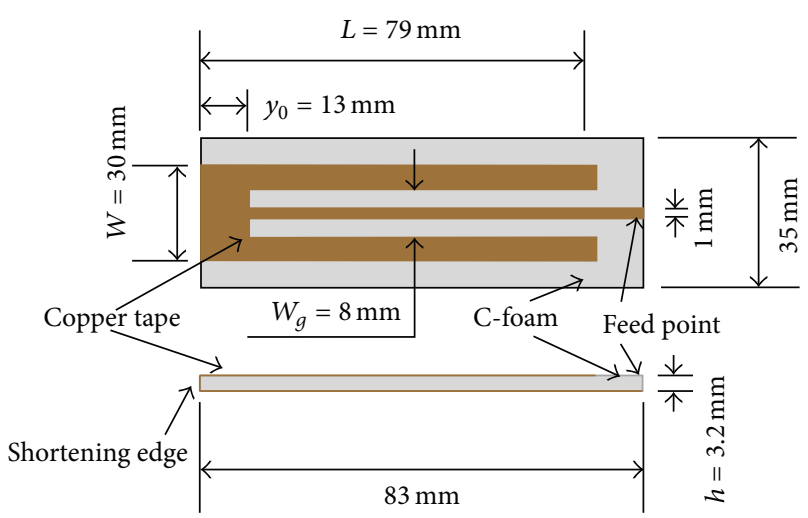

(b)

FIGURE 2: Structure of quarter-wave folded inset-fed rectangular patch antenna placed on silver fabric ground plane in three-dimensional view (a) and with dimensions (b).

as the effective width of the radiating elements would be severely reduced, yielding a degraded antenna performance. Instead, in an optimisation process, the width of the insetfeed line is reduced to $1 \mathrm{~mm}$. Considering the electric field distribution, the overall inset width, including the slots and feeding strip, is selected as $W_{g}=8 \mathrm{~mm}$. The resulting radiating elements have a width of $11 \mathrm{~mm}$ on each side.

By using a discrete voltage port rather than a microstrip line wave port in the simulations, we have also been able to show that the thin inset feed allowed the antenna to be fed using a balanced structure that can be directly connected to the two-port WISP chip output (see Section 4.4). The proposed antenna is modelled and simulated using CST Microwave Studio 2012 using a $78 \Omega$ discrete port to provide an approximate match to the input impedance of the unbalanced WISP chip.

The physical dimensions are intentionally chosen to be integer millimetre values to ensure that the antenna remains practicable and simpler to fabricate. The conductor used to construct the antenna is copper foil $(0.035 \mathrm{~mm}$ thick). A nonconductive adhesive attached to the foil allows a reliable construction of the antenna.

The power gain is defined as the product of the directivity and the radiation efficiency of the antenna. A directivity of $5-8 \mathrm{~dB}$ can be expected from the cavity model [16] for a rectangular microstrip patch. However, the actual values are expected to be severely affected by the limited size of the antenna ground plane as well as the close proximity of a human body as discussed later in Section 4. Therefore, to decouple the antenna from the effects of the human body while providing an extended ground plane, a silver fabric ground plane is added to the realized design. The RIPSTOP silver fabric sheet with dimensions of $200 \times 200 \mathrm{~mm}$ is selected for this purpose, because of its low sheet resistivity of less than $0.25 \Omega /$ sq.

3.2. Study of the Critical Design Parameters. This section considers the impact of various parameters on the resonance frequency and reflection coefficient $\left(S_{11}\right)$ in the operation band. These considerations are important, especially for an RFID

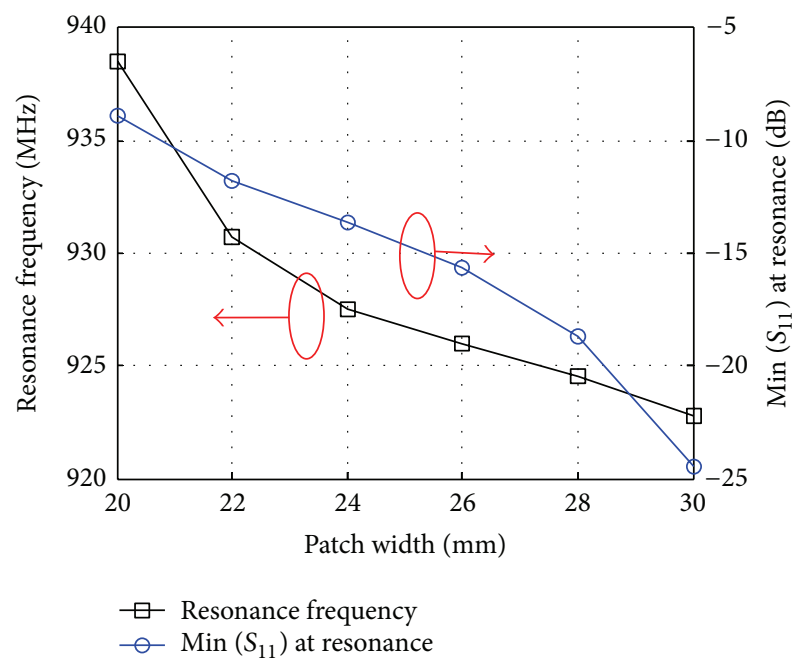

FIGURE 3: Simulated antenna resonant frequency and minimum reflection coefficient within a specific bandwidth versus variation of patch width $W$.

tag antenna designer to (i) select appropriate physical dimensions to optimise the performance of the design and (ii) to tune the final antenna once connected to the WISP. Our main focus is to achieve a high impedance match and to increase the gain of the antenna.

3.2.1. Width of the Radiation Patch. The width of the antenna patch $(W)$ controls the input impedance at the first edge of the patch and the radiation pattern. Figure 3 shows that with increasing patch width $W$, the antenna tends to resonate at lower frequencies and has a better impedance match (lower reflection coefficient) to the target input impedance for a fixed inset point. Hence, in order to maximise the match to the WISP's input impedance, the patch width is chosen to be the largest value permitted within the dimension restrictions (see Table 1).

3.2.2. Inset-Feed Position. Figure 4 shows the reflection coefficient according to the change in feed position. The antenna feed input impedance can be lowered by shifting the insetfeed position $y_{0}$ towards the folded edge. The minimum value 


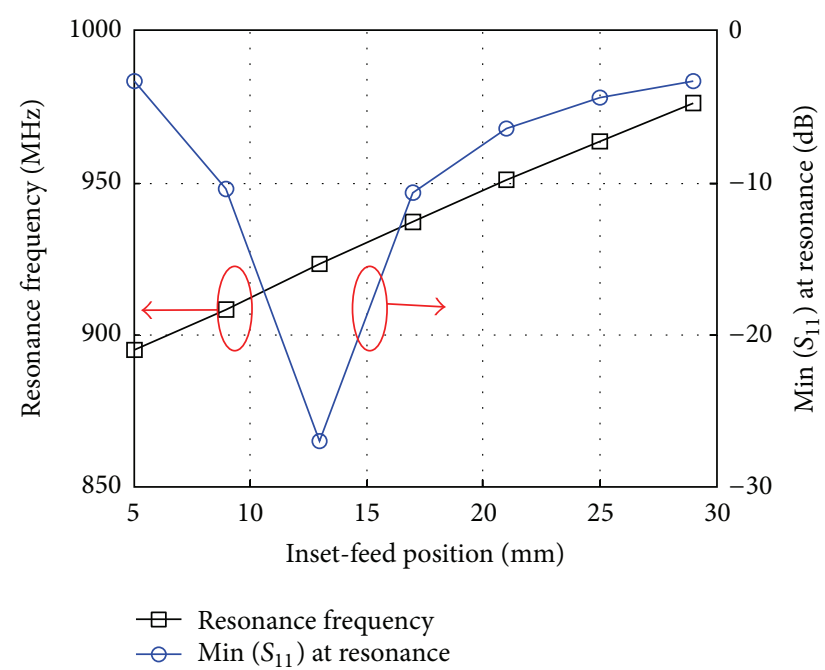

FIGURE 4: Simulated antenna resonant frequency and minimum reflection coefficient within the specified bandwidth versus variation of feed position $y_{0}$.

of reflection coefficient varies accordingly and attains a minimum at a specific distance $y_{0}=13 \mathrm{~mm}$ at the centre frequency of the operation bandwidth, at $923 \mathrm{MHz}$. Figure 4 also illustrates that the resonant frequency increases when the feed position is shifted away from the shorting edge of the patch.

3.2.3. Inset-Feed Slot Width. The reflection coefficient and the corresponding resonant frequency are also dependent on the width of the overall inset-feed width $W_{g}$. The fringing field distribution along the feed line can influence the radiating pattern as additional, orthogonally placed effective radiators. If the inset-feed slot is too wide, the antenna will radiate from these positions. If the width is too small, the fields of the travelling wave in the transmission line will couple into the microstrip resonator. It can be observed from the field distribution that the antenna starts to radiate through the inset-feed slots when they become larger than $8 \mathrm{~mm}$. This is manifested in Figure 5 as discontinuity in the curves.

\section{Impact of Human Body}

For the intended application of the device in human activity recognition, the antenna is by nature placed near a human body. Thus, the effects of human tissue in close proximity, such as skin or fat tissue, must be included in our considerations. In simulation models, the antenna is placed at distances of $d_{h}=3 \mathrm{~mm}, 10 \mathrm{~mm}$, and $20 \mathrm{~mm}$ from the human body model consisting of a $1 \mathrm{~mm}$ thick skin layer and $5 \mathrm{~mm}$ thick fat tissue layer. The dielectric constants chosen in this design are $\varepsilon_{r}=31.2$ for skin and $\varepsilon_{r}=4.60$ for fat, while the conductivities are $\sigma=0.585 \mathrm{~S} / \mathrm{m}$ for skin and $\sigma=$ $8.01 \mathrm{~S} / \mathrm{m}$ for fat.

For this investigation of the body effects, the standalone antenna, that is, without an extended ground plane, is modelled first. Afterwards, a $200 \times 200 \mathrm{~mm}$ layer of silver fabric is placed below the antenna to extend the ground plane and

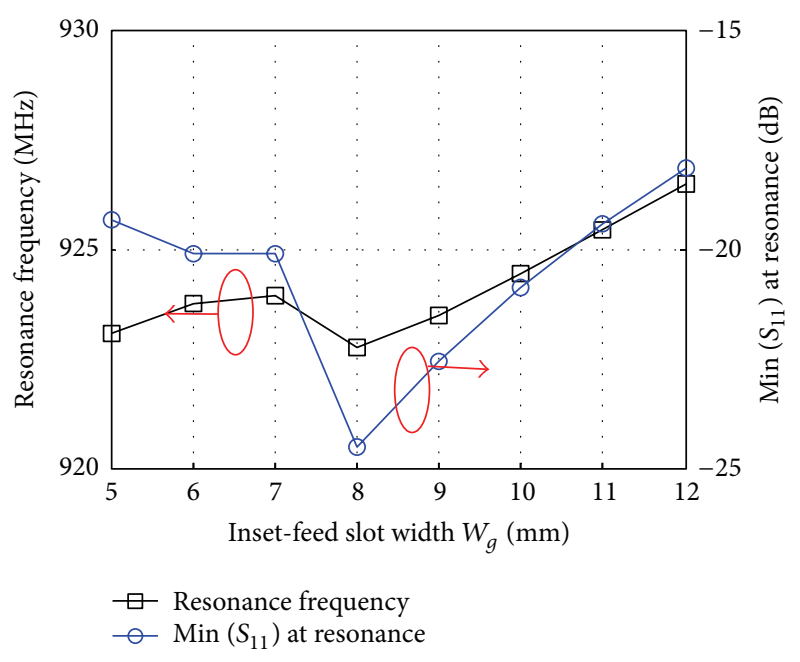

FIGURE 5: Simulated antenna resonant frequency and minimum reflection coefficient within the specified bandwidth versus variation of gap width.

reduce the impact of the human body. For each situation, the reflection coefficient and radiation patterns are compared.

4.1. Standalone Antenna without Silver Fabric Ground Plane. The reflection coefficient of the standalone antenna without the silver fabric is shown in Figure 6 in free space and in the vicinity of the human body model. A severe detuning can be observed, with a widening of the curve resonant dip suggesting large losses, when the human body is placed close to the body model. Figure 7 shows the radiation pattern of the simulated antenna in free space and at a distance of $3 \mathrm{~mm}$ to the tissue at $923 \mathrm{MHz}$. In free space, due to the very limited size of the ground plane, a gain of $1.3 \mathrm{dBi}$ is predicted from simulations. In the vicinity of the body, the antenna performance is significantly degraded and the maximum gain is decreased to $-2.1 \mathrm{dBi}$ as a consequence of the severe coupling into the human tissue.

4.2. Elimination of Human Body Attenuation Using a Silver Fabric Ground Plane. To minimise the attenuation caused by the human body as well as to increase both the gain and efficiency of the antenna, a large ground plane is added to this design. For this purpose, a sheet of flexible RIPSTOP silver fabric is attached to the bottom copper plane of the antenna. The use of silver fabric facilitates the integration of the antenna into clothing while maintaining the flexibility of the original design.

Figure 8 demonstrates the effectiveness of the sheet of silver fabric in eliminating the detuning and attenuation caused by a human body. The $S_{11}$ characteristics experience no noticeable variation when the antenna is placed in proximity of the human body model, and the overall performance is highly insensitive to the distance $d_{h}$ from the tissue model. The same statement can be made about the simulated radiation characteristics as shown in Figure 9, where the gain patterns are almost identical in both simulated $x y$ - and $x z$-principal planes. 


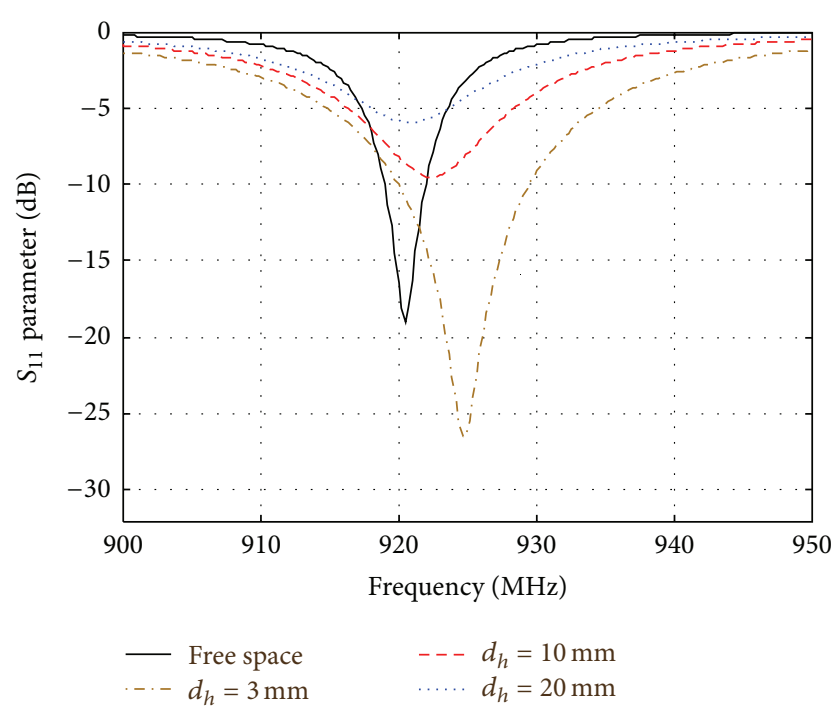

FIGURE 6: Simulated antenna (without silver fabric) reflection coefficient in free space at various distances from the human body.
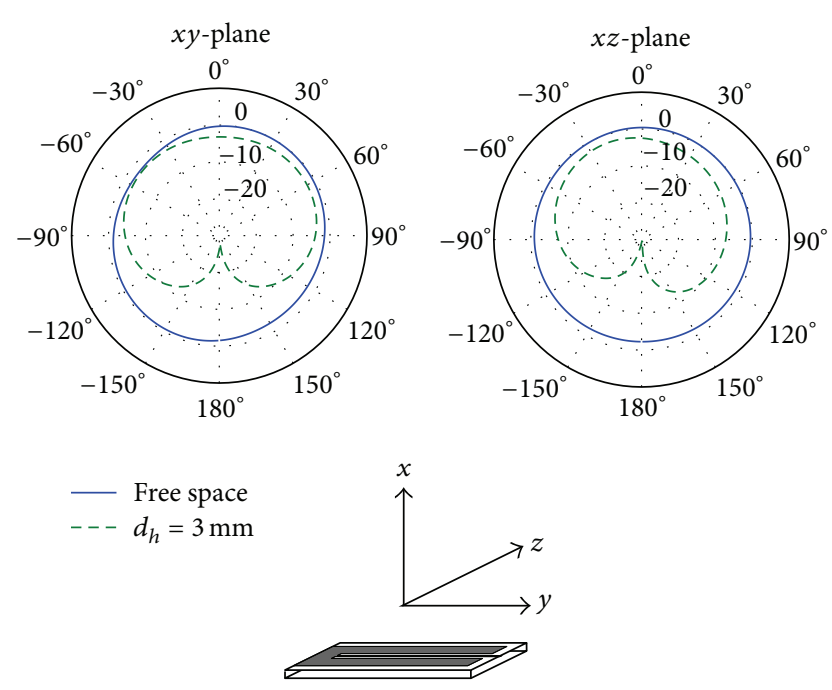

FIGURE 7: Simulated standalone antenna gain (without silver fabric) at a frequency of $923 \mathrm{MHz}$.

4.3. Final Design. Based on the parametric study in Section 3.2 and the results presented in this section, the antenna structure in Figure 1 is fabricated with a $200 \times 200 \mathrm{~mm}$ silver fabric ground plane. The free space simulation results in Figure 8 indicate that the antenna resonates at $f_{r}=$ 924.23 MHz and has an impedance bandwidth of $6.6 \mathrm{MHz}$ defined for $\left|S_{11}\right|<-10 \mathrm{~dB}$. The predicted maximum gain is $2.8 \mathrm{dBi}$ as shown in Figure 9, with an efficiency of around $60 \%$, where the losses can be mainly attributed to the imperfect conductivity of the silver fabric.

4.4. Feeding the Antenna with a WISP. The connection from the two ports of the WISP chip to the antenna is realised using parallel metallic wires. This is illustrated in Figure 10 and functions as narrow band balun. The effect of the exact geometry of this arrangement on the resonant frequency and minimum reflection coefficient is investigated

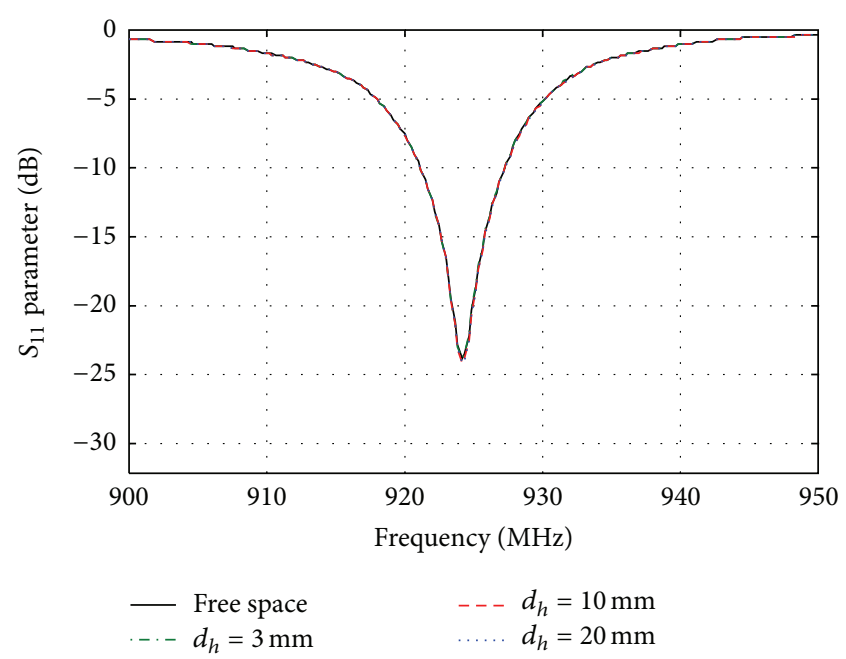

FIGURE 8: Simulated antenna (with silver fabric) reflection coefficient in free space and for various distances to the human body.
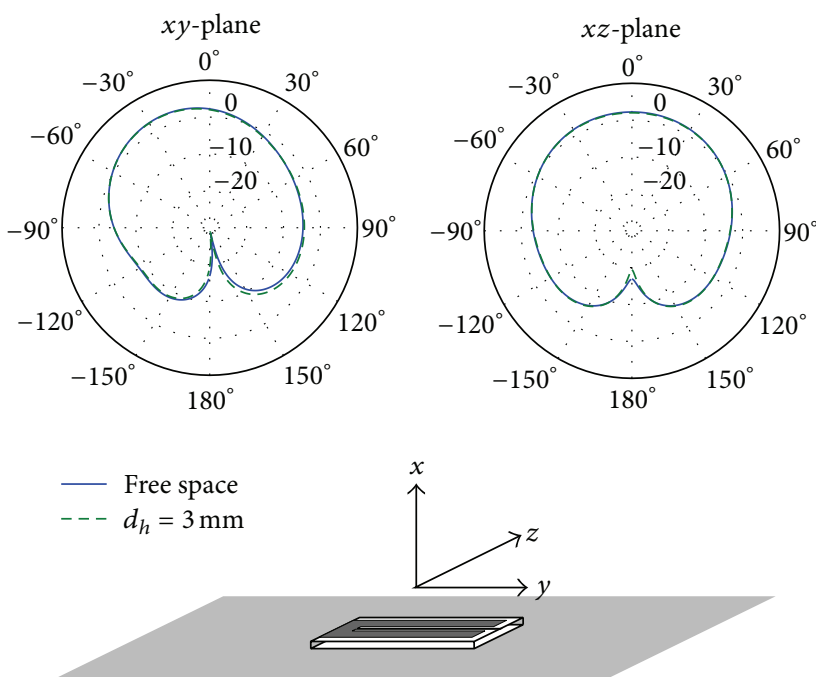

FIgURE 9: Simulated gain of the final design (with silver fabric) at a frequency of $923 \mathrm{MHz}$.

through a parametric study. The two parameters of interest are the radius $r$ and length $l$ of the balanced paired-wire transmission line. The simulated range for the radius is $r=$ $[0.025,0.05,0.1,0.25,0.5] \mathrm{mm}$ and the wire length is $l=$ $[0,2.5,5, \ldots, 17.5,20] \mathrm{mm}$.

Figure 11 shows variations of the resonance frequency as a function of the wire length $l$. It can be observed that longer wires cause a detuning towards lower frequencies. This effect is more pronounced with thinner wires. To further illustrate the effect, the maximum reflection coefficient within the specified frequency band is plotted in Figure 12 and indicates a change in the bandwidth. The results again show that for wire lengths longer than $l=5 \mathrm{~mm}$, the connection method is unsuitable, and even more so for thin wires. Figure 12 also shows that an optimum length of $l=5 \mathrm{~mm}$ yields an acceptable return loss for all radii. Therefore, this wire length $l=5 \mathrm{~mm}$ and a radius of $0.1 \mathrm{~mm}$ are selected to connect the antenna to the WISP chip. 


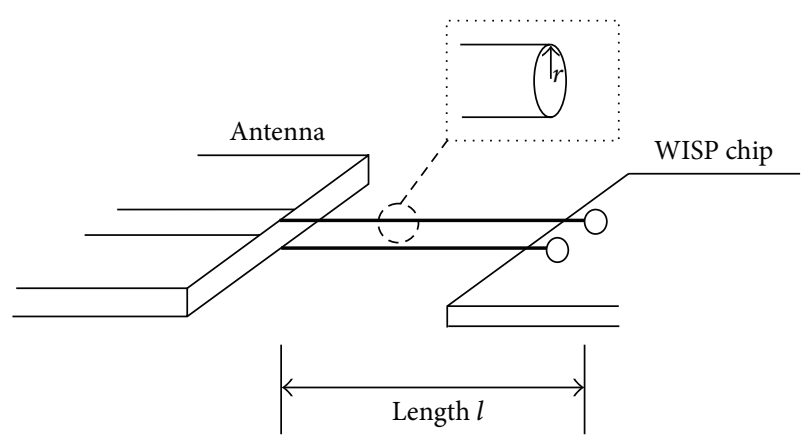

FIgURE 10: Connection between the antenna and the WISP chip.

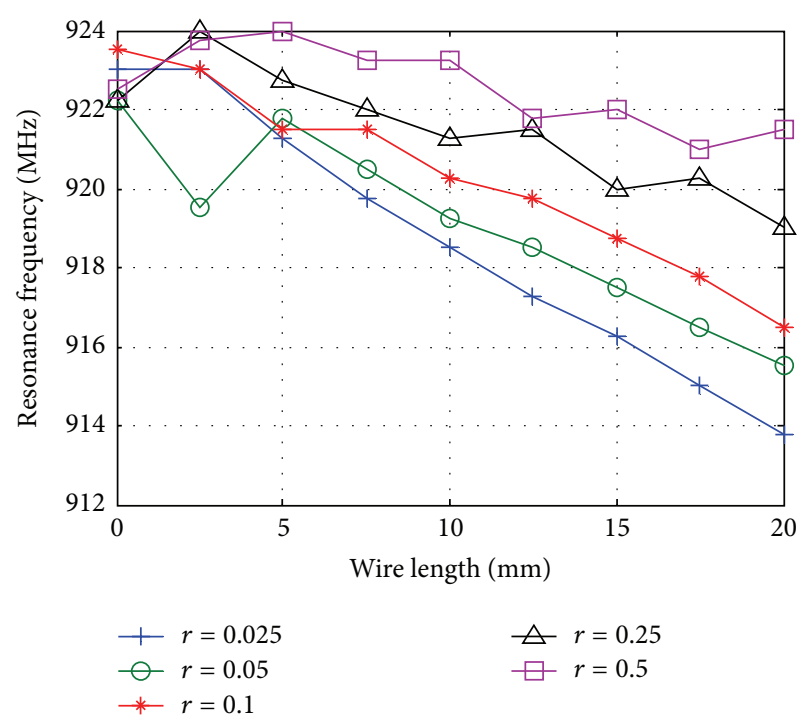

FIGURE 11: Simulated resonance frequency for different wire lengths $l$ and radius $r$.

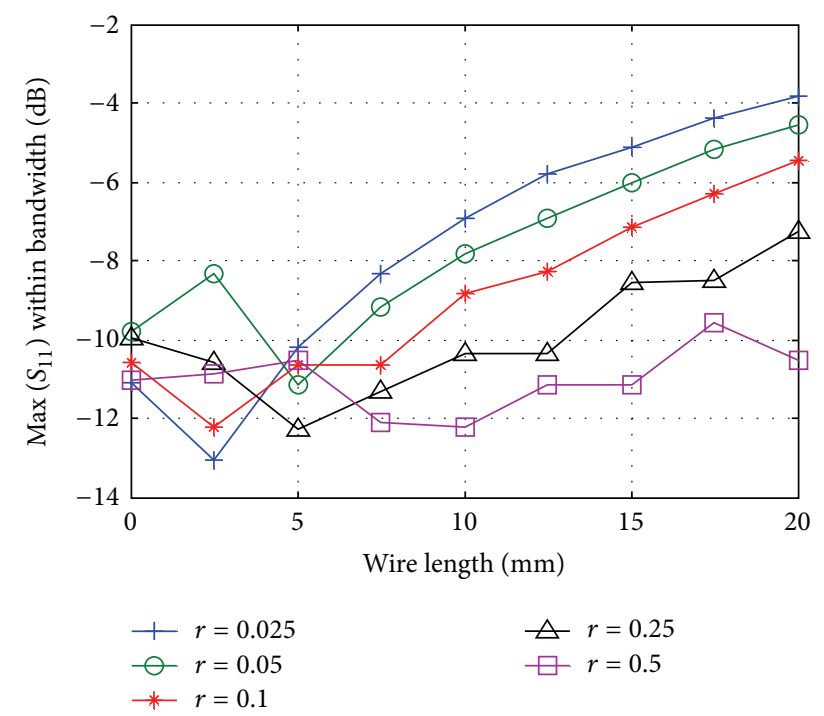

FIGURE 12: Simulated maximum reflection coefficient within the specified bandwidth from 920 to $926 \mathrm{MHz}$ for different wire lengths $l$ and radius $r$.

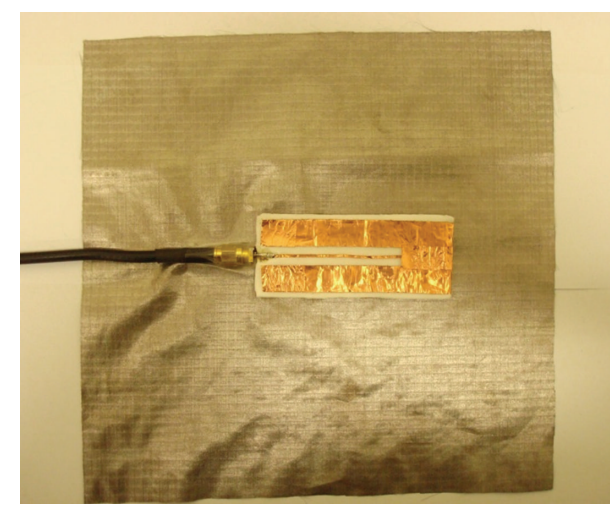

FIGURE 13: Fabricated antenna with SMA connector for impedance measurements.

\section{Experimental Results}

This section describes the measured results obtained from a manufactured antenna prototype and compares them with the predictions from simulations presented in Section 4. The experiments include measurement of the antenna input impedance, reflection coefficient, RSSI, read rate, and read range tests. The performance testing of the WISP [2] has been carried out first in an anechoic box and then worn on a human subject in a practical operating environment (laboratory).

5.1. Antenna Reflection Coefficient. Two similar antennas have been fabricated and the measurements of reflection coefficient were carried out by connecting the fabricated antenna to a vector network analyser using a $50 \Omega$ SMA connector, as shown in Figure 13. The validity of measuring the reflection coefficient for determining the resonance frequency and bandwidth using a connector of slightly different impedance has been confirmed in separate simulations. During the measurement phase, the antenna was tuned by progressively adding copper tape such that the inset-feed point was eventually placed at $y_{0}=14 \mathrm{~mm}$ from the shorting edge. This tuning step was necessary to meet resonance at $923 \mathrm{MHz}$, that is, at the centre frequency in the $920-926 \mathrm{MHz}$ band. Figure 14 shows the measured reflection coefficient of the two antennas and $-10 \mathrm{~dB}$ bandwidths are listed in Table 2.

Comparing the two manufactured antennas, Antenna 1 (see Table 2) shows a better reflection coefficient and has been chosen for connection to the WISP chip. In the original version, the WISP chip is connected to a dipole. So, the original dipole was removed as illustrated in Figure 15. The WISP was then soldered onto the folded microstrip patch antenna using two short wires. The device with WISP chip connected to the proposed folded patch antenna is shown in Figure 16. The silver fabric was attached using conductive epoxy.

5.2. Wearable WISP Tag Performance in Anechoic Box. The next set of experiments compares the performance of the $\mathrm{W}^{2}$ ISP, that is, the wearable WISP with the inset-fed microstrip patch structure, to the performance of the original WISP including a half-wave dipole structure. In the setup for these 


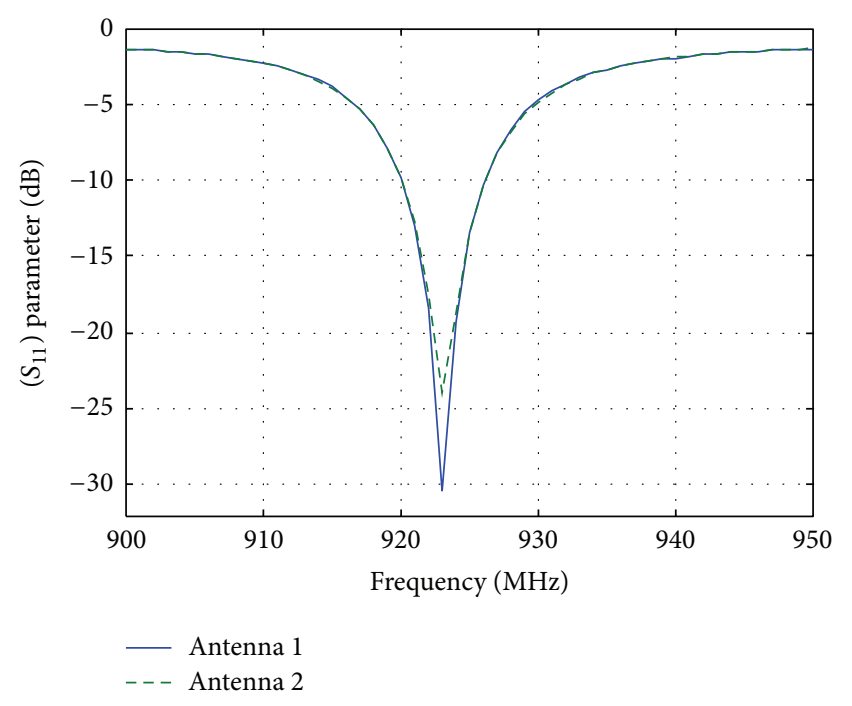

FIGURE 14: Measured reflection coefficient of fabricated antennas with silver fabric ground plane.

TABLE 2: Measured $-10 \mathrm{~dB}$ bandwidth of fabricated antennas.

\begin{tabular}{lccc}
\hline Antenna No. & $\begin{array}{c}f_{r} \\
(\mathrm{MHz})\end{array}$ & $\begin{array}{c}-10 \mathrm{~dB} \mathrm{BW} \\
(\mathrm{MHz})\end{array}$ & $\begin{array}{c}-10 \mathrm{~dB} \text { Band } \\
(\mathrm{MHz})\end{array}$ \\
\hline Antenna 1 & 923.07 & 6.07 & $920.04-926.11$ \\
Antenna 2 & 923.11 & 6.10 & $920.07-926.17$ \\
\hline
\end{tabular}

measurements, a circular right-hand circularly polarised (RHCP) reader antenna with a gain of $G_{t}=6 \mathrm{dBi}$ has been used. Considering that the tag antennas are horizontally polarised, a polarisation loss factor of $L_{p}=3 \mathrm{~dB}$ has to be taken into account in the power budget calculation. Using the simulated gain, the calculated maximum read ranges in free space based on the Friis transmission equation (3) are $r=4.7 \mathrm{~m}$ for the folded microstrip antenna and $r=4.4 \mathrm{~m}$ for the dipole antenna. The estimated read range for the dipole antenna assumes no coupling onto the human body and the performance can potentially be severely degraded due to losses in the human body. In a practical complex indoor multipath environment, these read ranges can be further reduced in worst case scenarios.

An Impinj Speedway R420 RFID reader was used for obtaining the RFID performance metrics. The frequency range was set to Australian UHF ISM (industrial-scientificmedical) band used for RFID ( $920 \mathrm{MHz}$ to $926 \mathrm{MHz}$ ). The WISP was activated by the interrogation signal received from the Impinj reader [1].

For comparison, all of the measurements of the dipole and the proposed folded microstrip antenna have been performed under identical environmental conditions, that is, placed in an anechoic box at the same distance from the reader, as shown in Figure 17. This ensures the consistency of the results since both devices are affected by the same environmental factors, such as reflections and interference from other equipments operating in the same band.

The first measured performance indicator is the RSSI, described by (1). RSSI values were measured at distances from

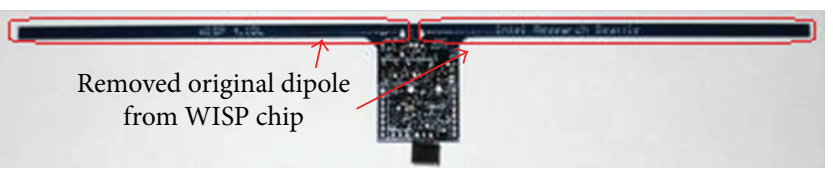

FIGURE 15: The original dipole is cut off from the WISP and the new folded microstrip antenna is connected to the balanced port in the centre. The length of the original dipole is $145 \mathrm{~mm}$.

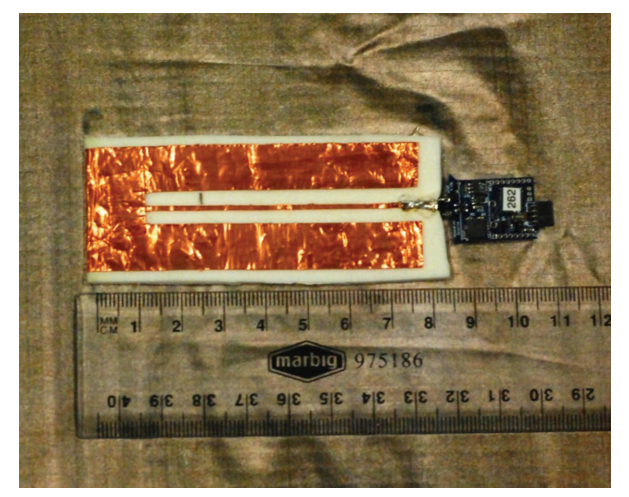

FIGURE 16: WISP connected to the proposed antenna. The silver fabric is attached using conductive epoxy.

0.5 meter to 2 meters for both the dipole antenna and the wearable folded microstrip patch antenna without a human body in its vicinity. Comparison of the RSSI values displayed in Figure 18 clearly scales with the higher maximum gain of the wearable antenna design and the results indicate that the proposed wearable antenna receives on average $1 \mathrm{~dB}$ stronger signals than the dipole. These results correspond well with the expected results, as the simulations predict a gain of $2.1 \mathrm{~dB}$ for the dipole versus a gain of $2.8 \mathrm{~dB}$ for the wearable folded microstrip antenna. It is emphasized that this slight gainrelated improvement of the performance is achieved in free space configuration and is only a small contribution to overall more significant improvement achieved when worn on a body. In addition, the main beam of the wearable microstrip antenna is pointed slightly upwards with its maximum at approximately 30 degrees elevation (see Figure 11). This is a desirable characteristic for the human activity monitoring application since the reader antennas are likely to be mounted on the ceiling.

Figure 19 shows the read rates (see Section 2.2) of the two antennas at different transmitted power levels. Again, the tests have been performed using the experimental arrangement shown in Figure 17, while using different transmit power levels at a distance of 1 meter. The proposed wearable antenna consistently provides better read rates at all power levels. The minimum reader transmit power at which the wearable WISP could be read is at $19 \mathrm{dBm}$, while the dipole-based WISP stopped being activated below $22 \mathrm{dBm}$. The $\mathrm{W}^{2}$ ISP is successfully read by the reader using a lower signal power and consequently a longer read range is expected.

Using the experimental setup in Figure 17, the performance of the tag in different directions has been measured to ensure satisfactory tag read performance. The read rate 


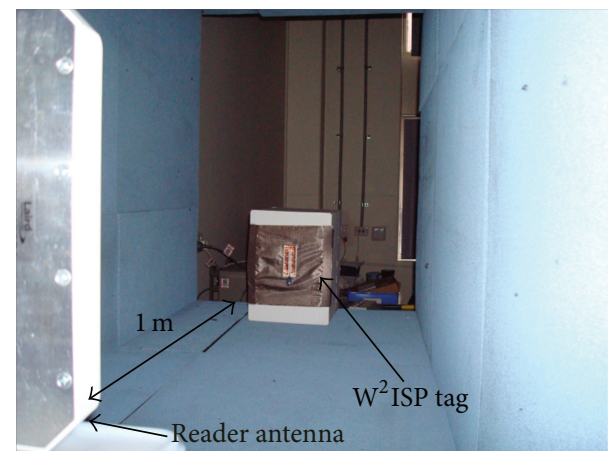

Figure 17: Anechoic box experimental arrangement.

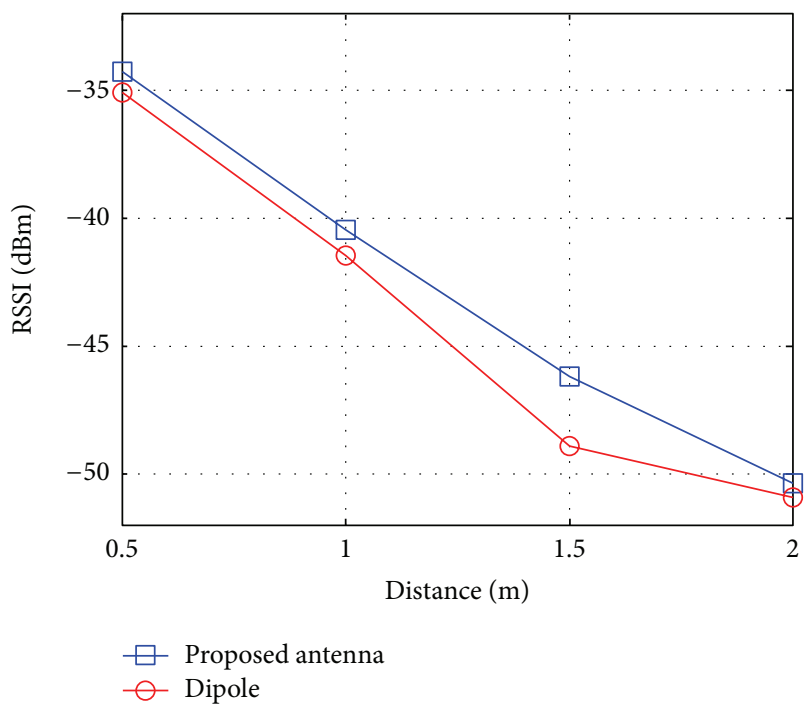

FIGURE 18: Measured RSSI versus distance in the anechoic box without a human body in the vicinity.

shown in Figure 20 is a good indicator of the gain of the antenna. When varying the orientation of the antenna with respect to the reader in \pm 90 degree angular range, the proposed folded microstrip antenna achieves consistently higher read rates than the original dipole. The slight reduction in read rate at an angle of 0 degree can be explained through multipath in the imperfectly anechoic measurement environment.

5.3. Wearable WISP Tag Performance on a Human Body. Measurements were performed to compare the performance of the wearable WISP when worn over clothing by a human subject, including the isolating silver fabric extended ground as shown in Figure 21, and compared to the performance of the original dipole. In order to reduce the detuning of the dipole, a polystyrene isolator with a thickness of $10 \mathrm{~mm}$ is used as shown in Figure 22.

The read range measurements were performed under real-world conditions in a laboratory room at different distances using a transmit power of $P_{t}=1, \mathrm{~W}=30 \mathrm{dBm}$. It can be observed from Figure 23 that the read range performance of the proposed wearable antenna is significantly improved

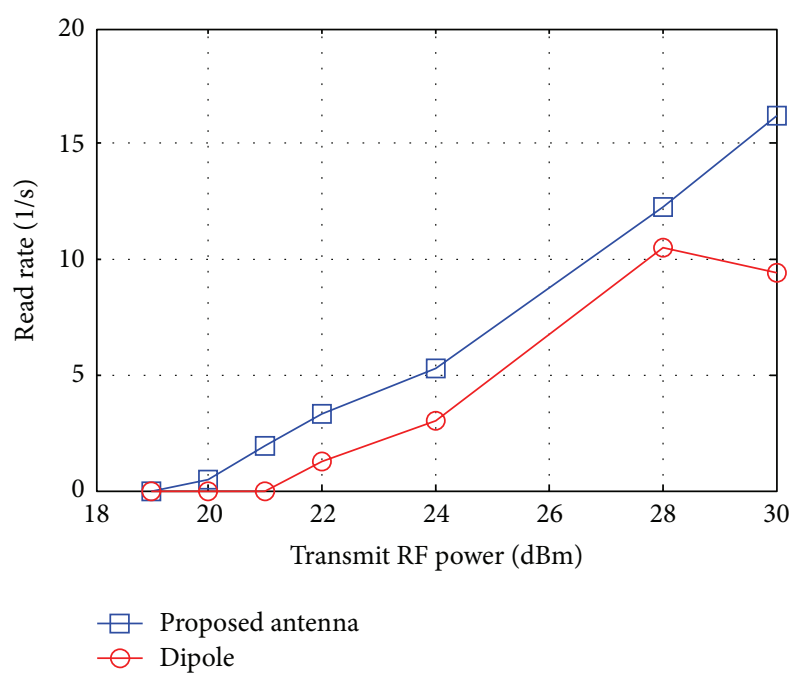

FIGURE 19: Measured read rates versus transmitted power at a fixed distance of 1 meter in the anechoic box without a human body close by.

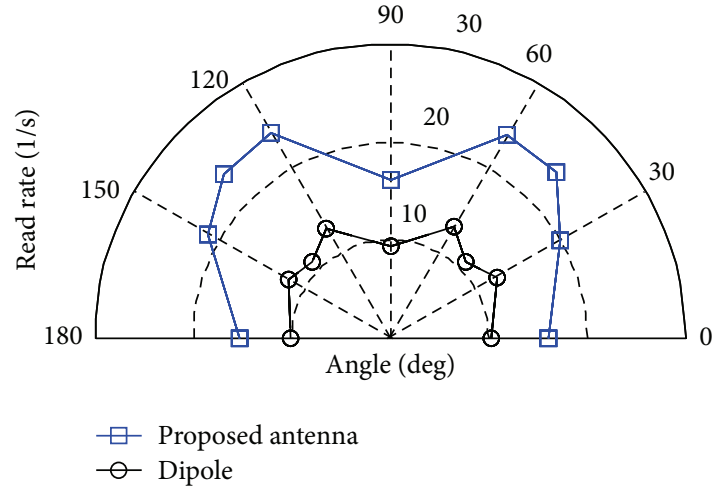

FIGURE 20: Measured read rates versus antenna facing angle in the anechoic box.

from the original dipole-based WISP. The proposed antenna meets all the requirements outlined in Table 1. The original WISP with the dipole antenna fails to power the WISP beyond $2.5 \mathrm{~m}$. This degraded performance compared to the $4.4 \mathrm{~m}$ estimated from the Friis transmission equation (3) is expected because of the losses arising from coupling of the dipole antenna into the human body. The WISP with the new wearable folded microstrip patch antenna continues to be read up to a maximum read range of $4 \mathrm{~m}$. These results are in good agreement with the theoretical maximum read range of $4.7 \mathrm{~m}$ calculated with the Friis transmission equation (3).

\section{Conclusion}

In this paper, a new implementation of a wearable, flexible antenna for the RFID sensor platform WISP has been proposed. The specifications require the whole system to be small and comfortable enough to be worn over a long period of time in the chest area for human activity recognition, for example, fall detection in elderly care. 


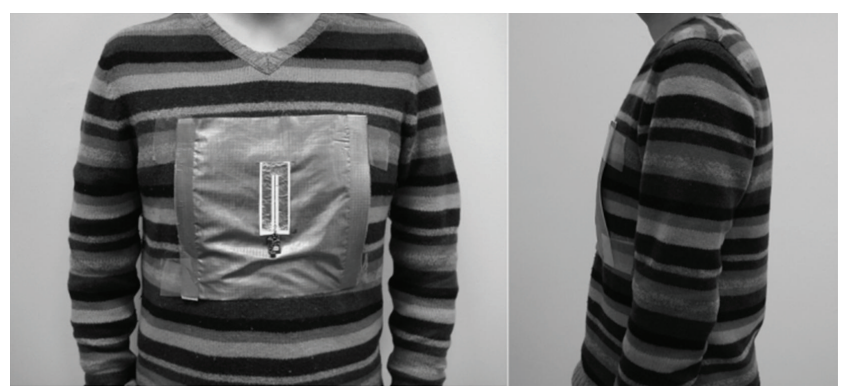

Figure 21: Proposed folded microstrip antenna worn on human body.

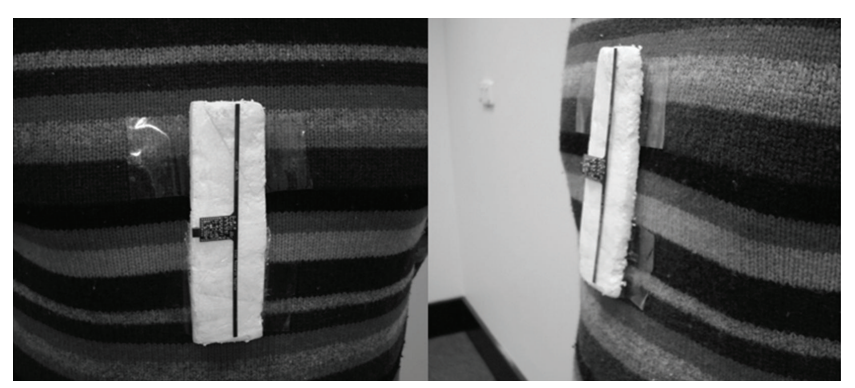

Figure 22: Dipole antenna worn on human body. The thickness of the polystyrene is about $10 \mathrm{~mm}$, which leaves enough space from human body to the WISP tag.

The main features of the new antenna are its low-profile, its flexibility, and a reliable read range. The simulated and experimental results demonstrate that the proposed design satisfies all the $\mathrm{W}^{2}$ ISP system requirements. The following features can be concluded for this antenna design.

(1) The folded microstrip implementation allows to reduce the size of the antenna to only half that of the original dipole design.

(2) An inset feed instead of a coaxial line feed can simplify the manufacturing process and improve the ruggedness of the antenna, especially when using a flexible substrate.

(3) The tag is low profile and flexible, and therefore, it is suitable for wearable applications.

(4) With the sheet of silver fabric, the detuning and attenuation arising from the human body can be minimised while the gain of the antenna is improved.

(5) Comparing to the original dipole, the read rate of the antenna is increased while the size is reduced. The 4-meter read range achieved satisfies current experimental requirements of the WISP application $[1,2]$.

Based on this design, further studies will be conducted with the goal of improving the usability in a real-world environment.

\section{Acknowledgments}

This research was supported by The Hospital Research Foundation (THRF) and the Australian Research Council

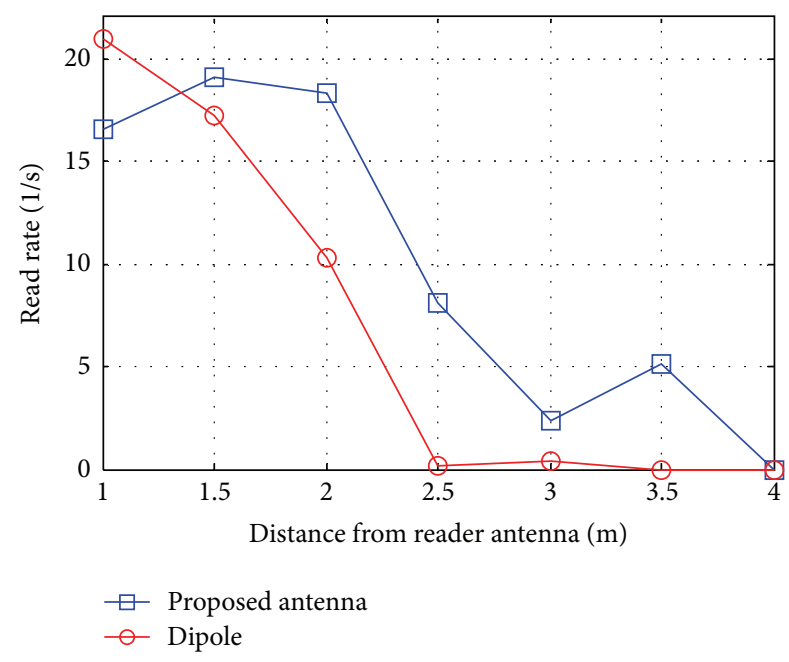

FIGURE 23: Measured read rates in the laboratory room with the antenna being worn on a human body.

(DP130104614). C. Fumeaux acknowledges the support by the Australian Research Council DP120100661. The authors would like to thank Professor P.H. Cole at the University of Adelaide for his support and guidance and Dr. J. R Smith and A. P. Sample at the University of Washington for their support of this research project.

\section{References}

[1] D. C. Ranasinghe, R. L. S. Torres, A. P. Sample, J. R. Smith, K. Hill, and R. Visvanathan, "Towards falls prevention: a wearable wireless and battery-less sensing and automatic identification tag for real time monitoring of human movements," in Proceedings of the 34th Annual International IEEE EMBS Conference, 2012.

[2] R. Visvanathan, D. C. Ranasinghe, and R. L. S. Torres, "Framework for preventing falls in acute hospitals using passive sensor enabled radio frequency identification technology," in Proceedings of the 34th Annual International IEEE EMBS Conference, 2012.

[3] B. H. Calhoun, J. Lach, J. Stankovic et al., "Body sensor networks: a holistic approach from silicon to users," Proceedings of the IEEE, vol. 100, no. 1, pp. 91-106, 2012.

[4] M. Kangas, A. Konttila, I. Winblad, and T. Jämsä, "Determination of simple thresholds for accelerometry-based parameters for fall detection," in Proceedings of the 29th Annual International Conference of IEEE-EMBS, Engineering in Medicine and Biology Society (EMBC '07), pp. 1367-1370, Lyon, France, August 2007.

[5] A. K. Bourke, J. V. O’Brien, and G. M. Lyons, "Evaluation of a threshold-based tri-axial accelerometer fall detection algorithm," Gait and Posture, vol. 26, no. 2, pp. 194-199, 2007.

[6] T. E. Lockhart, A. T. Barth, X. Zhang, R. Songra, E. Abdel-Rahman, and J. Lach, "Portable, non-invasive fall risk assessment in end stage renal disease patients on hemodialysis," in Proceedings of the 1st Wireless Health Conference (WH '10), pp. 84-93, October 2010.

[7] A. P. Sample, D. J. Yeager, P. S. Powledge, A. V. Mamishev, and J. R. Smith, "Design of an RFID-based battery-free programmable 
sensing platform," IEEE Transactions on Instrumentation and Measurement, vol. 57, no. 11, pp. 2608-2615, 2008.

[8] M. Marroncelli, D. Trinchero, V. Lakafosis, and M. M. Tentzeris, "Concealable, low-cost paper-printed antennas for WISP-based RFIDs," in Proceedings of the 5th IEEE International Conference on RFID (RFID '11), pp. 6-10, April 2011.

[9] A. Rida, L. Yang, R. Vyas, and M. M. Tentzeris, "Conductive inkjet-printed antennas on flexible low-cost paper-based substrates for RFID and WSN applications," IEEE Antennas and Propagation Magazine, vol. 51, no. 3, pp. 13-23, 2009.

[10] K. V. S. Rao, P. V. Nikitin, and S. F. Lam, "Antenna design for UHF RFID tags: a review and a practical application," IEEE Transactions on Antennas and Propagation, vol. 53, no. 12, pp. 3870-3876, 2005.

[11] B. A. Sani, M. Rajab, R. Foster, and Y. Hao, "Antennas and propagation of implanted RFIDs for pervasive healthcare applications," Proceedings of the IEEE, vol. 98, no. 9, 2010.

[12] L. Mo and C. Qin, "Tunable compact UHF RFID metal tag based on cpw open stub feed PIFA antenna," International Journal of Antennas and Propagation, vol. 2012, Article ID 167658, 8 pages, 2012.

[13] H. Attia, M. M. Bait-Suwailam, and O. M. Ramahi, "Enhanced gain planar inverted-F antenna with metamaterial superstrate for UMTS applications," in Proceedings of the Progress in Electromagnetics Research Symposium (PIERS '10), vol. 6, pp. 585-588, July 2010.

[14] R. Li, G. DeJean, M. M. Tentzeris, and J. Laskar, "Development and analysis of a folded shorted-patch antenna with reduced size," IEEE Transactions on Antennas and Propagation, vol. 52, no. 2, pp. 555-562, 2004.

[15] P. V. Nikitin, R. Martinez, S. Ramamurthy, H. Leland, G. Spiess, and K. V. S. Rao, "Phase based spatial identification of UHF RFID tags," in Proceedings of the 4th Annual IEEE International Conference on RFID (RFID '10), pp. 102-109, April 2010.

[16] C. A. Balanis, Antenna Theory: Analysis and Design, John Wiley \& Sons, New York, NY, USA, 2005. 

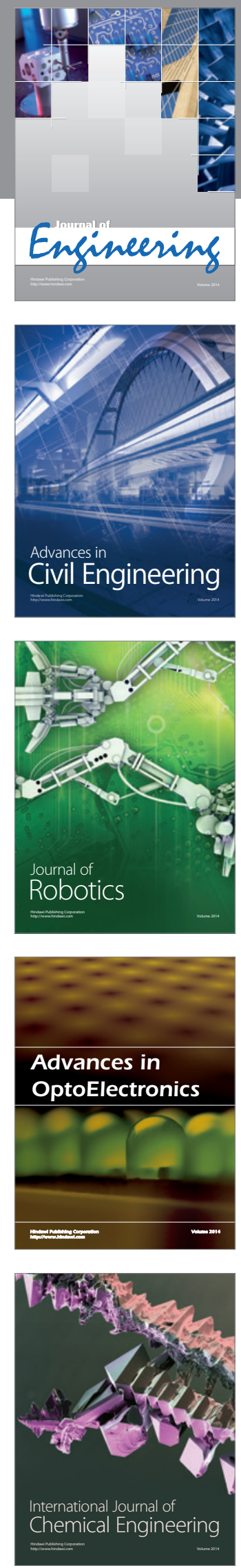

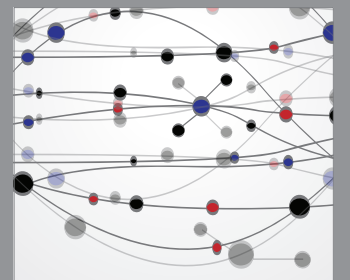

The Scientific World Journal
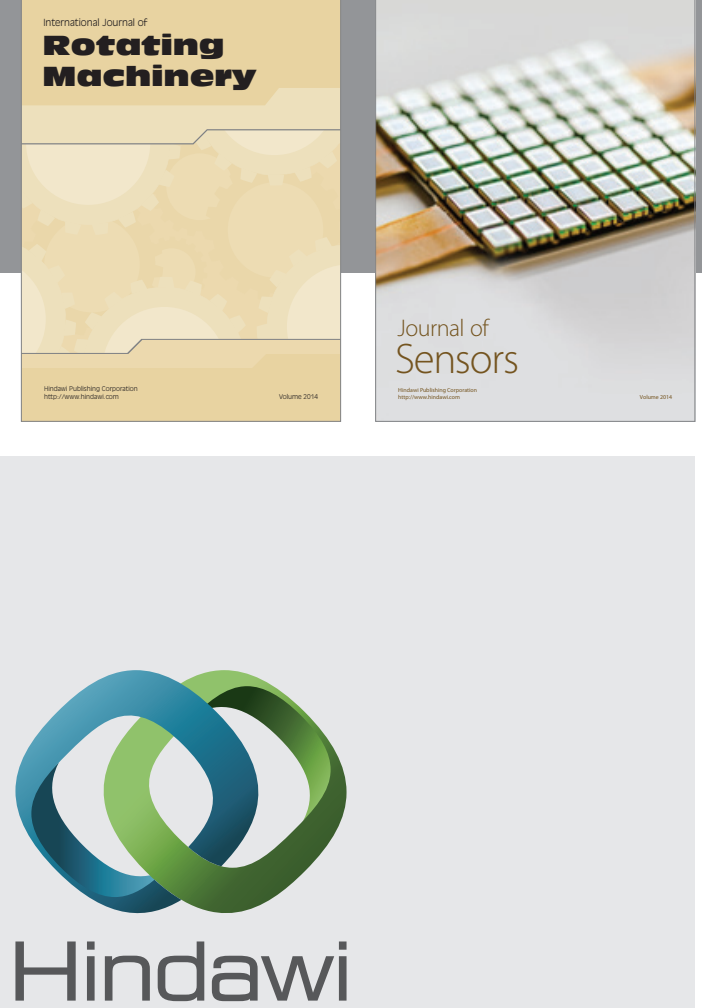

Submit your manuscripts at http://www.hindawi.com
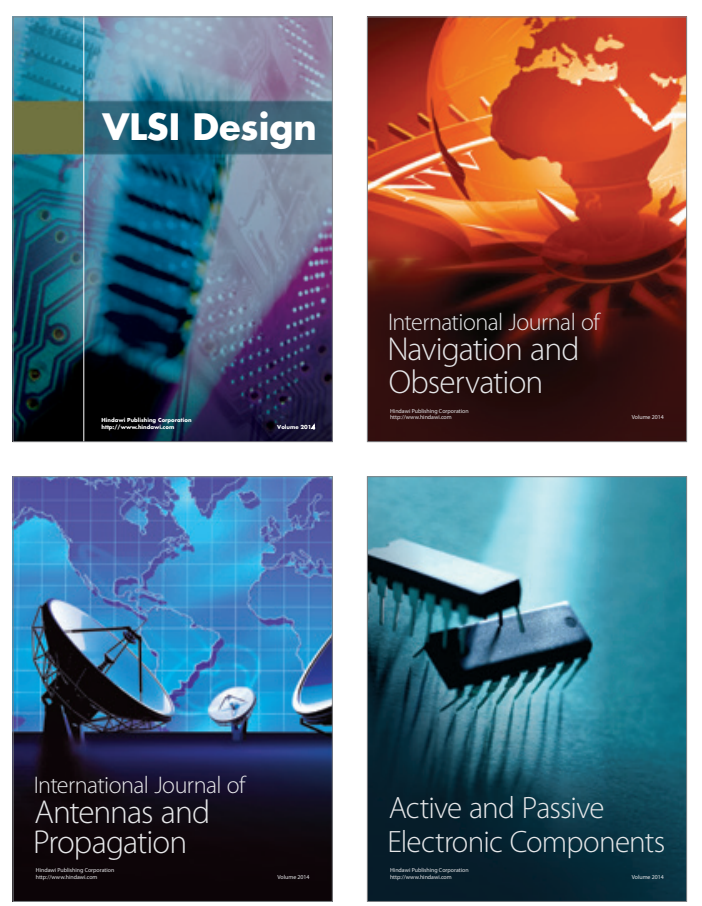
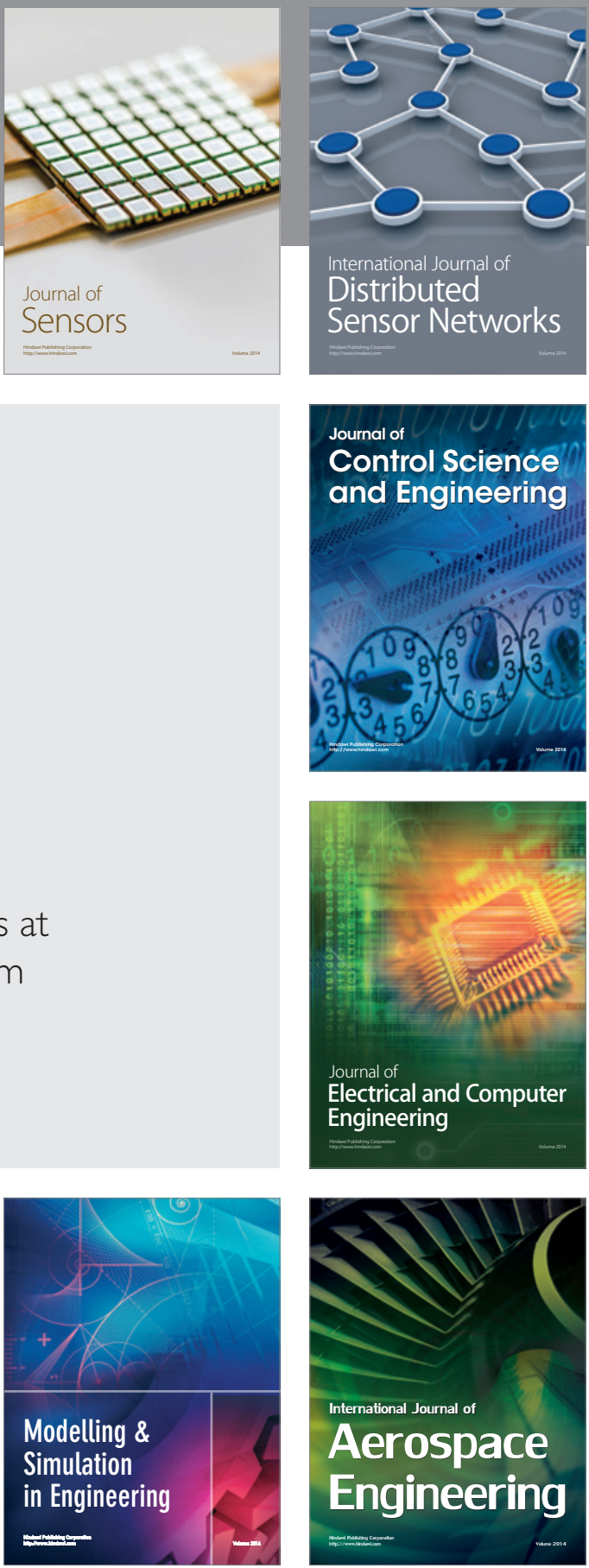

Journal of

Control Science

and Engineering
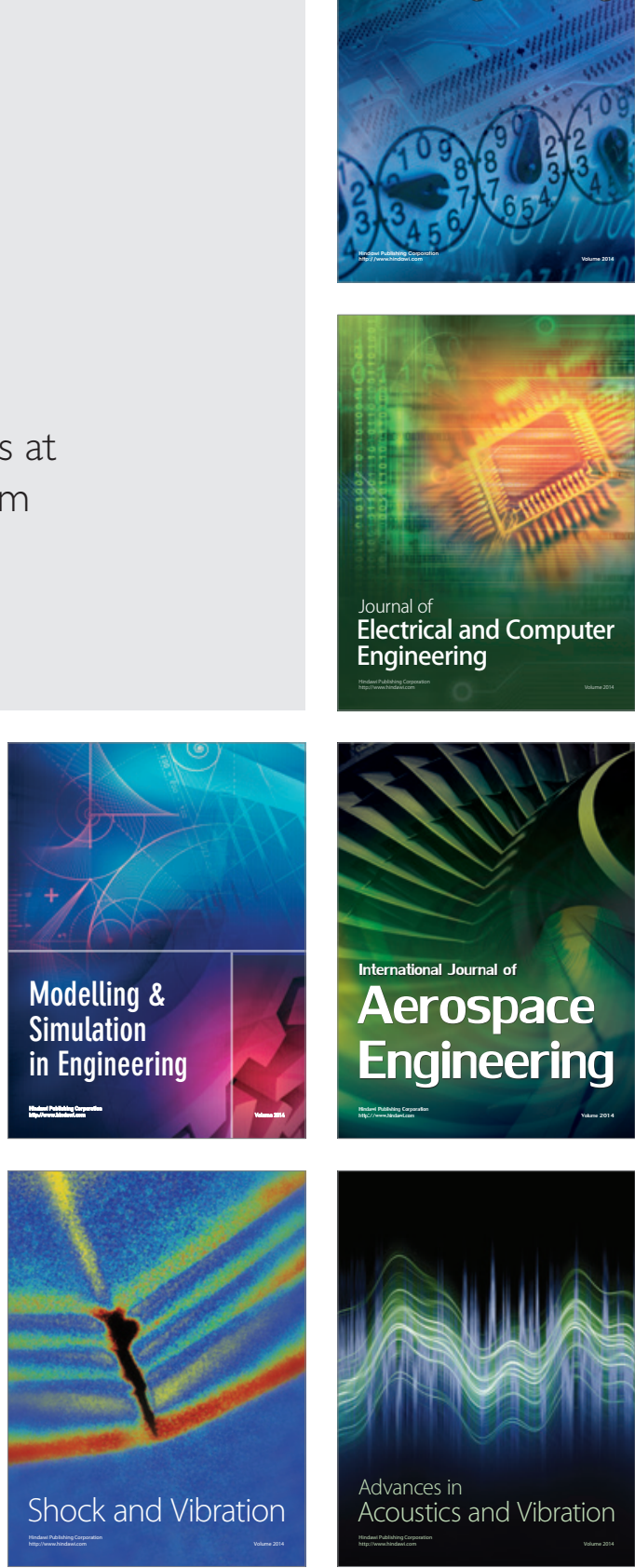